\title{
Unlicensed assisted transmission in vehicular edge computing networks
}

\author{
Zhongbao $\mathrm{Ji}^{1}$, Xiao Lu², Rui Yin ${ }^{3^{*}}$ (1) and Celimuge Wu${ }^{4}$
}

\author{
${ }^{*}$ Correspondence: \\ yinrui@zucc.edu.cn \\ ${ }^{3}$ School of Information \\ and Electrical Engineering, \\ Zhejiang University City \\ College, Hangzhou, China \\ Full list of author information \\ is available at the end of the \\ article
}

\begin{abstract}
Recently, the emerging computation-intensive in-vehicle applications and the exponentially growing data have posed a serious challenge to resource-limited in-vehicle devices. By integrating the mobile edge computing (MEC) into the vehicle networks, vehicular edge computing (VEC) is envisioned as a promising solution. In this paper, we divide the VEC system into small time slots, which effectively simulates dense small-scale computing scenarios in the rapidly varying channel environment over time. Furthermore, to alleviate the shortage of spectrum resources, vehicles can offload part of computing tasks to the edge server not only through licensed channels but also through unlicensed channels. However, dynamic topology changes and complex communication characteristics of the vehicle networks lead to the strategies of task offloading and resource allocation being difficult to obtain. By decoupling the non-convex optimizing problem and splitting it into multiple sub-problems, we design a joint power, spectrum and computing resource allocation strategy with low complexity, in order to minimize the total energy consumption of all vehicles for processing tasks. Theoretical analysis and simulation results validate the effectiveness of the proposed scheme that employing licensed and unlicensed access in VEC networks can reduce the energy consumption of vehicles for processing tasks in comparison with baseline schemes.
\end{abstract}

Keywords: Mobile edge computing, Vehicular edge computing, Task offloading, Resource allocation, Unlicensed channels

\section{Introduction}

With the rapid development of urbanization and intelligence, intelligent transportation system (ITS) has received increasing attention from industry and academia [1-3]. One of the key components of ITS is the vehicle networks [4]. In the face of exponentially growing data and some emerging computation-intensive in-vehicle applications such as autonomous driving [5], augmented reality (AR) technology [6], traditional vehicle networks are being severely challenged in terms of communication, computation and storage.

The vehicular cloud computing services, which combine cloud computing and vehicle networks, allow vehicles to send data to the cloud for calculation and storage $[7,8]$. It is undeniable that the emerging architecture fulfils the above-mentioned author(s) and the source, provide a link to the Creative Commons licence, and indicate if changes were made. The images or other third party material in this article are included in the article's Creative Commons licence, unless indicated otherwise in a credit line to the material. If material is not included in the article's Creative Commons licence and your intended use is not permitted by statutory regulation or exceeds the permitted use, you will need to obtain permission directly from the copyright holder. To view a copy of this licence, visit http:// creativecommons.org/licenses/by/4.0/. 
communication and computing needs, but it also comes at the cost of non-negligible transmission latency.

In line with the new trend of computing moving from clouds to network edges over the last decade [9], vehicular edge computing (VEC) has been proposed [10]. By integrating the mobile edge computing (MEC) into the vehicle networks, VEC harvests idle computation power and storage space distributed at the edge of the vehicle networks and significantly reduces communication overhead and transmission latency [11].

Different from traditional MEC, VEC is characterised by its highly mobile vehicles, which leads to more dynamic topology changes and more complex communication characteristics [12]. In this particular scenario, the strategies of task offloading and allocation of computational and communication resources become more challenging.

Although the US Federal Communications Commission (FCC) has allocated 75 $\mathrm{MHz}$ of licensed spectrum in the $5.9 \mathrm{GHz}$ band for dedicated short-range communications (DSRC) [13, 14], these spectrum resources will become scarce as the number of connected vehicles and the supporting infrastructure increases rapidly. On the basis, unlicensed spectrum resources, with the feature of free access, can be a powerful complement to licensed spectrum resources. Therefore, it is of practical importance for the research on employing licensed and unlicensed access in VEC networks.

In this paper, we introduce additional unlicensed spectrum to expand the spectrum resources available to vehicles and to provide more flexible combinations for offloading tasks. Besides, we develop a joint power, spectrum and computing resource allocation strategy to minimize the total energy consumption of all vehicles for processing tasks. The main contributions of this paper are summarized as follows:

- Both licensed and unlicensed spectrums are considered in the system because of their advantages and disadvantages. Compared with the scarce licensed spectrum resources, the unlicensed spectrum can be accessed for free. However, as a cost of more freedom on computation offloading, vehicles need to share unlicensed channels with WiFi users and consume extra energy to sense the state of the unlicensed channels before transmission. As a matter of course, offloading on the unlicensed spectrum becomes a powerful complement to that on the licensed spectrum. Our novel consideration of integrating licensed and unlicensed spectrum for simultaneous offloading has great research value and practical significance.

- The system divided by small time slots effectively simulates dense small-scale computing scenarios under the rapidly varying channel environment over time. By decoupling the non-convex objective function and constraints, the optimization problem of minimizing total energy consumption for processing tasks is split into multiple sub-problems. Furthermore, a low-complexity algorithm is proposed to realize the optimal allocation of computing and communication resources.

- Extensive simulation results validate the effectiveness of the proposed scheme that employing licensed and unlicensed access in VEC networks can reduce the energy consumption of vehicles for processing tasks in comparison with baseline schemes. Moreover, we evaluate the impacts of different parameters and vehicular environments on the proposed scheme and offer valuable guidelines for real deployment. 
The rest of the paper is organized as follows. In Sect. 2, some related works are reviewed and discussed. The aim, methods and settings of the study are described in Sect. 3. In Sect. 4, we illustrate the system model and formulate the optimization problem. A lowcomplexity algorithm is proposed to realize the optimal allocation of computing and communication resources in Sect. 5. In Sect. 6, simulation results are presented to verify the efficiency of the proposed scheme. Finally, Sect. 7 concludes the paper.

\section{Related work}

In recent years, many researchers have been engaged in optimizing the resource allocation of MEC systems to minimize the energy consumption of task processing while ensuring latency requirements [15-20]. In [15], task offloading in MEC has been formulated as a stochastic optimization problem, with the objective of minimizing the energy consumption of task offloading while guaranteeing the average queue length. An online joint radio and computational resource management algorithm for multi-user MEC systems has been proposed in [16], with the objective of minimizing the long-term average weighted sum power consumption of the mobile devices and the MEC server, subject to a task buffer stability constraint. Under the goal of minimizing long-term energy consumption, the authors in [17] have imposed the probabilistic and statistical constraints task queue lengths to guarantee latency requirements at short timescales. The authors in [18] have decomposed the task offloading process into three phases, i.e. task uploading, task executing and result downloading, and have developed a low-complexity algorithm to solve the three-stage weighted sum energy consumption minimization problem. An air-ground integrated multi-access edge computing system has been studied. Then, an online deep reinforcement learning scheme has been proposed to approximate the Q-factor, which is used to maximize the average utility of mobile users in [19]. In [20], a reinforcement learning algorithm is based on end-edge-cloud collaboration to find proper routes in a proactive manner with low communication overhead.

To address the tension between computation-intensive and latency-sensitive in-vehicle applications and resource-limited in-vehicle devices, a significant amount of research work has focused on the integration of MEC and vehicular networks in recent years [21-25]. The authors in [21] have proposed a solution that enables vehicles to learn the offloading delay performance of their neighbouring vehicles while offloading computation tasks. On the basis, a distributed adaptive learning-based task offloading (ALTO) algorithm based on the multi-armed bandit theory has been designed to minimize the average offloading delay without requiring frequent state exchange. The authors in [22] have estimated the transmission rates for the vehicle to vehicle and vehicle to infrastructure communication based on practical assumptions, and have obtained the optimal task allocation scheme subject to the maximum tolerable delay and vehicle's stay time. In [23], both the communication and computation states have been modelled by finite Markov chains and the task scheduling and resource allocation strategy have been solved jointly to maximize users' Quality of Experience (QoE). The authors in [24] have proposed a hybrid intelligent optimization algorithm, which not only determines where the tasks are performed, but also indicates the execution order of the tasks on the server. In [25], a new computing paradigm, called by Collaborative Vehicular Edge Computing (CVEC), has been proposed to maximize user-centric utility, which aims to incentivize 
parked vehicles to contribute free resources to collaborate with MEC servers on computing tasks.

With the rapid growth of wireless devices, the available licensed spectrum resources are becoming increasingly scarce. Therefore, many works are devoted to improve spectrum utilization [26-30]. To improve sensing and transmission performance of the cognitive Industrial Internet of Things (IIoT), a cluster-based the cognitive IIoT has been proposed in [26] with the goal of maximizing the average total throughput wherein the cluster heads perform cooperative spectrum sensing to get available spectrum, and the nodes transmit via non-orthogonal multiple access (NOMA). In [27], a multibeam satellite IIoT in Ka-band has been proposed to realize wide-area coverage and long-distance transmissions, which uses NOMA for each beam to improve transmission rate. The authors in [28] have combined Internet of Things (IoT) with fifth generation (5G) network to expand the communication resources of the IoT and have explored the tradeoff between energy consumption and transmission rate in different scenarios. In [29], a 5G-based green broadband communication system has been proposed to combine wireless information transfer (WIT) and wireless power transfer (WPT) and a joint optimization unit has been deployed to maximize the system throughput by jointly optimizing subband sets and subband powers subject to the constraints of energy requirement and interference. A novel market-based solution has been proposed in [30] for co-channel interference management in multibeam satellite systems (MSS) by introducing an elastic price mechanism.

In contrast to improving the efficiency of spectrum utilization in vehicular networks, some researchers aim to expand the available spectrum resources by utilizing unlicensed spectrum through spectrum sharing techniques [31-34]. In [31], a context-aware communication has been proposed to approach to efficiently integrate different licensed and unlicensed spectrum leveraging the MEC. With the goal of maximizing the number of active cellular vehicle-to-everything (V2X) users, an energy sensing-based spectrum sharing scheme has been designed in [32], where cellular V2X users and vehicular ad hoc network (VANET) users are able to access the unlicensed channels fairly. The authors in [33] have proposed a heterogeneous vehicular network, where V2X users can share licensed spectrum with cellular users and unlicensed spectrum with WiFi users, and have formulated an optimization problem to maximize the spectrum efficiency of V2X communications while guaranteeing the quality of services (QoS) requirements of cellular users and WiFi users. In [34], the authors have constructed an intelligent offloading framework for 5G-enabled vehicular networks, which allocates licensed and unlicensed spectrum resources to cellular vehicle-to-infrastructure (V2I) users and vehicle-to-roadside unit (V2R) users, respectively. Then, a deep-reinforcement-learning-based method has been investigated to solve the cost minimization problem by considering the latency constraint of users.

However, the advantages and disadvantages of licensed and unlicensed spectrum in VEC networks have not been adequately addressed in the related works. On the other hand, as in-vehicle devices continue to evolve, employing licensed and unlicensed access in VEC networks can be expected. Based on this, the research on simultaneous offloading via licensed and unlicensed spectrum in VEC networks is still lacking, which is the contribution of this work. 


\section{Methods}

In this paper, we study a single-cell multi-vehicle time-division VEC system, in which one road-side unit (RSU) is linked to one MEC server through the fibre-wired link and provides computing services for the vehicles within its coverage area. Considering the dynamic topological changes resulting from the high mobility of the vehicles, we partition the system into small time slots. Based on the assumption that vehicles are running independent and fine-grained tasks, small-scale computational tasks arrive at the beginning of each time slot and are processed within the current time slot. Moreover, vehicles are free to allocate the proportions of three types of task processing, including local computing, offloading via licensed channels and offloading via unlicensed channels.

By decoupling the non-convex objective function and constraints, the optimization problem of minimizing total energy consumption for processing tasks is split into multiple sub-problems. Ultimately, a joint power, spectrum and computing resource allocation strategy with low complexity has been devised to minimize the total energy consumption of all vehicles for processing tasks within each time slot.

The effectiveness of the proposed scheme is supported by the experimental evaluation, while all experiments are performed on MATLAB R2020a running on a Windows 64 machine with $2.9 \mathrm{GHz}$ CPU and $16 \mathrm{~GB}$ RAM.

\section{System model and problem formulation}

\subsection{System model}

As depicted in Fig. 1 we consider a single-cell multi-vehicle VEC system, where one RSU is linked to one MEC server through the fibre-wired link and provides computing services for the vehicles within its coverage area. Each resource-limited vehicle often needs to offload computational tasks to the MEC server, in order to reduce the

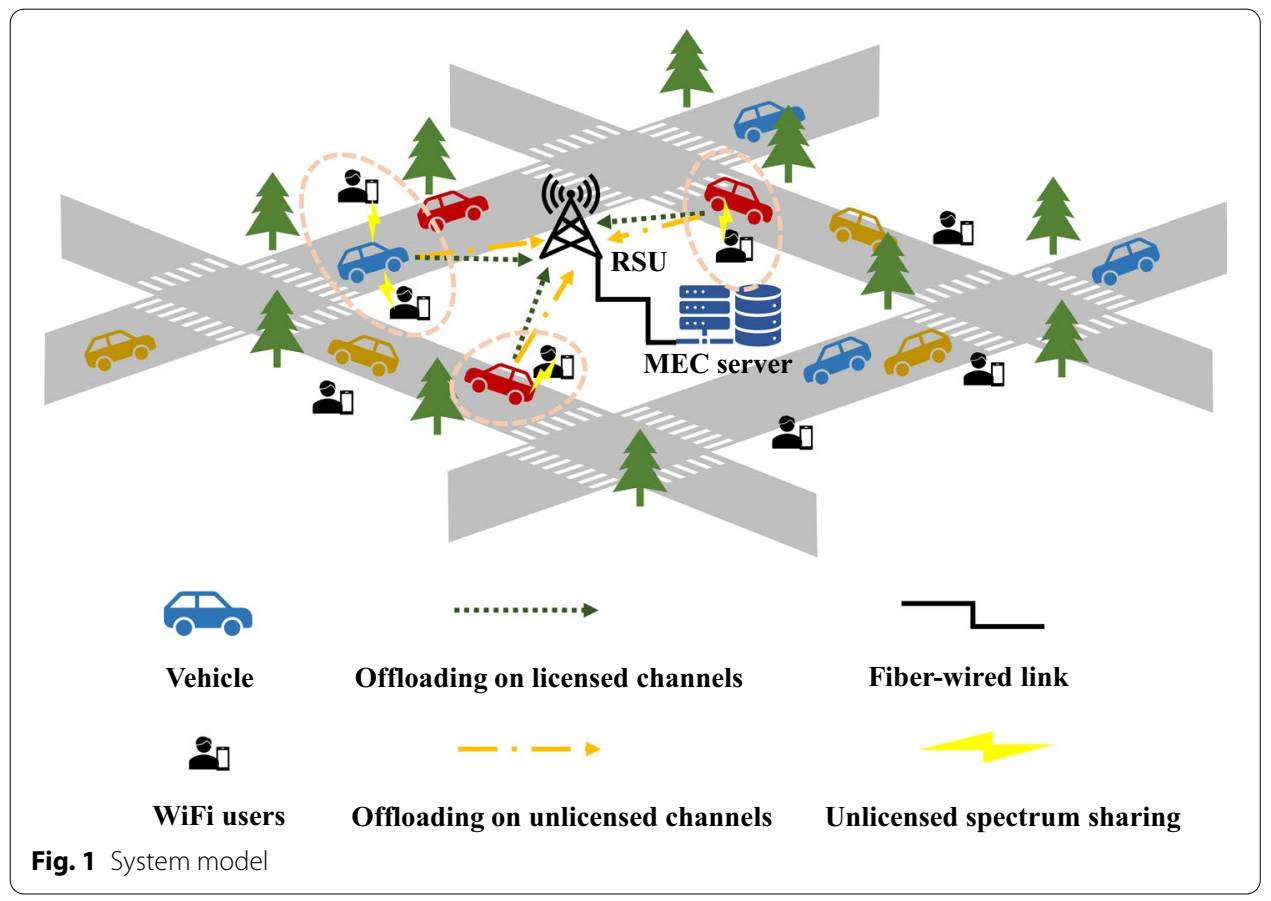


cost of processing computation-intensive tasks, such as latency or energy consumption. The set of vehicles can be denoted as $\mathcal{K}=\{1,2, \ldots, K\}$. As computing resources are plentiful on the MEC server, we ignore the cost of processing tasks from the vehicles on the MEC server and focus on the trade-off between local computing and offloading to the MEC server.

Considering the scarcity of licensed spectrum resources, the vehicles can communicate wirelessly with the RSU and transmit computational tasks via unlicensed spectrum. On the one hand, unlicensed spectrum can be accessed for free, which brings more freedom for offloading. In the VEC systems without unlicensed spectrum, vehicles have to cope with the pressure of computing tasks by increasing the transmit power and the local CPU frequency, which results in significant energy consumption. On the other hand, the feature of free-to-use on unlicensed spectrum may cause serious channel collision. On this basis, access by vehicles will have an impact on the rest of the users of the unlicensed band, such as WiFi users. To mitigate this collision, vehicles need to consume extra energy to sense the state of the unlicensed channels before transmission. Moreover, when the licensed band is allocated orthogonally to the vehicles using frequency division multiple access (FDMA), the duty-cycle muting (DCM) mechanism allows vehicles and WiFi users to share unlicensed bands in the time division multiple access (TDMA) manner.

The system operates in a time-division manner. The length of each time slot is denoted as $\tau$ and let $\mathcal{T}=\{1,2, \ldots\}$ be the set of time slot indices. In the paper, we assume that vehicles are running independent and fine-grained tasks. The number of bits on the task arriving and being processed at time slot $t$ is denoted by $A_{k}(t)$. Without loss of generality, we assume that $A_{k}(t)$ in different time slots are independent and identically distributed (i.i.d.) and uniformly distributed in the interval $\left[0, A_{k}^{\max }\right], \forall k \in \mathcal{K}$.

\subsection{Computing model}

For local computing, the required CPU cycles to process one bit of computation task input from vehicle $k$ can be given as $M_{k}(t)$. Note that $M_{k}(t)$ depends on the types of applications and can be obtained by off-line measurements. Hence, the number of tasks, which can be calculated locally by vehicle $k$ at time slot $t$, is given by

$$
D_{k}^{\text {local }}(t)=\tau f_{k}(t)\left[M_{k}(t)\right]^{-1},
$$

where $f_{k}(t)$ is the CPU frequency of vehicle $k$ at time slot $t$ which cannot exceed its maximum value $F_{k}$.

According to circuit theories [35, 36], the energy consumption for local computation at vehicle $k$ is given by

$$
E_{k}^{\text {local }}(t)=\tau \mu_{k}\left(f_{k}(t)\right)^{3},
$$

where $\mu_{k}$ is the effective switched capacitance of the CPU at vehicle $k$, and it is related to the chip architecture [35]. 


\subsection{Communication model}

\subsubsection{Communication model on licensed channel}

To avoid co-channel interference, the licensed spectrum is allocated orthogonally to the vehicles via FDMA. According to the Shannon-Hartley formula [37], the offloaded bits at vehicle $k$ on the licensed channel are given by

$$
D_{k}^{\mathrm{L}}(t)=\psi_{k}(t) B^{\mathrm{L}} \tau \log _{2}\left(1+\frac{p_{k}^{\mathrm{L}}(t) h_{k}^{\mathrm{L}}(t)}{N_{0}^{\mathrm{L}} \psi_{k}(t) B^{\mathrm{L}}}\right) \text {, }
$$

where $\psi_{k}(t)$ is the proportion of licensed bandwidth allocated to vehicle $k$ under the constraint of $0 \leq \sum_{k=1}^{K} \psi_{k}(t) \leq 1, p_{k}^{\mathrm{L}}(t)$ denotes the transmit power of vehicle $k$ which cannot exceed its maximum value $P_{k}^{\mathrm{L}}, B^{\mathrm{L}}$ is the bandwidth of the licensed channel, $N_{0}^{\mathrm{L}}$ is the power spectrum density of the noise on the licensed channel, and $h_{k}^{\mathrm{L}}(t)$ is the channel gain between vehicle $k$ and the RSU in time slot $t$.

The energy consumption of vehicle $k$ on task offloading via licensed channel is given by

$$
E_{k}^{\mathrm{L}}(t)=p_{k}^{\mathrm{L}}(t) \tau
$$

\subsubsection{Communication model on unlicensed channel}

Although unlicensed spectrum provides additional spectrum resources and more flexible options for offloading computing tasks, it introduces more severe channel collisions. In this case, the DCM mechanism allows each vehicle to compete with the surrounding WiFi users for the utilization of unlicensed spectrum resource in a TDMA manner [38, 39]. Furthermore, based on the novel WiFi traffic load definition proposed in [40], each vehicle can estimate the maximum proportion of time it can use by sensing the state of the unlicensed channel before transmission to ensure fair co-existence with WiFi users.

Accordingly, the offloaded bits from vehicle $k$ on the unlicensed channel at time slot $t$ are given by

$$
D_{k}^{\mathrm{U}}(t)=s_{k}(t) B_{k}^{\mathrm{U}} \tau \log _{2}\left(1+\frac{p_{k}^{\mathrm{U}}(t) h_{k}^{\mathrm{U}}(t)}{N_{0}^{\mathrm{U}} B_{k}^{\mathrm{U}}}\right),
$$

where $s_{k}(t)$ is the time fraction occupied by vehicle $k$ on the unlicensed channel in time slot $t$ and $p_{k}^{\mathrm{U}}(t)$ denotes the transmit power on the unlicensed channel, which cannot exceed their maximum values, $S_{k}$ and $P_{k}^{\mathrm{U}}$, respectively; $B_{k}^{\mathrm{U}}$ is the bandwidth of the unlicensed channel associated with vehicle $k, N_{0}^{\mathrm{U}}$ is the noise power spectrum density on the unlicensed channel, and $h_{k}^{\mathrm{U}}(t)$ is the unlicensed channel power gain between vehicle $k$ and the RSU.

According to the regulatory rules of unlicensed channels, to avoid the collision, all users need to sense the state of the unlicensed channels before using them. Therefore, the energy consumption for channel sensing and task offloading on the unlicensed channel is given by

$$
E_{k}^{\mathrm{U}}(t)=p_{k}^{\mathrm{U}}(t) s_{k}(t) \tau+p_{k}^{\mathrm{S}}\left(1-s_{k}(t)\right) \tau,
$$


where $p_{k}^{S}$ is the power consumption of vehicle $k$ on sensing the unlicensed channel during the "off" period, which is a constant related to the device sensor architecture.

\subsection{Problem formulation}

In this sub-section, the optimization problem of computation offloading and resource allocation is designed for the novel VEC system with licensed and unlicensed access. This problem seeks to minimize the total energy consumption of all vehicles for processing tasks, under the constraints of limited computing and communication resources.

We denote the system operation as $\mathbf{X}(t) \triangleq\left\{\mathbf{f}(t), \mathbf{p}^{\mathrm{L}}(t), \mathbf{p}^{\mathrm{U}}(t), \mathbf{s}(t), \boldsymbol{\psi}(t)\right\}$, where $\quad \mathbf{f}(t) \triangleq\left\{f_{1}(t), f_{2}(t), \ldots, f_{K}(t)\right\}, \quad \mathbf{p}^{\mathrm{L}}(t) \triangleq\left\{p_{1}^{\mathrm{L}}(t), p_{2}^{\mathrm{L}}(t), \ldots, p_{K}^{\mathrm{L}}(t)\right\}$, $\mathbf{p}^{\mathrm{U}}(t) \triangleq\left\{p_{1}^{\mathrm{U}}(t), p_{2}^{\mathrm{U}}(t), \ldots, p_{K}^{\mathrm{U}}(t)\right\}, \quad \mathbf{s}(t) \triangleq\left\{s_{1}(t), s_{2}(t), \ldots, s_{K}(t)\right\}, \quad$ and $\psi(t) \triangleq\left\{\psi_{1}(t), \psi_{2}(t), \ldots, \psi_{K}(t)\right\}$. The sum energy consumption minimization problem can be formulated as follows:

$$
\begin{aligned}
& \min _{\mathbf{X}(t)} \quad \sum_{k \in \mathcal{K}}\left(E_{k}^{\text {local }}(t)+E_{k}^{\mathrm{L}}(t)+E_{k}^{\mathrm{U}}(t)\right) \\
& \text { s.t. } \quad 0 \leq f_{k}(t) \leq F_{k}, \forall t \in \mathcal{T}, k \in \mathcal{K}, \\
& 0 \leq p_{k}^{\mathrm{L}}(t) \leq P_{k}^{\mathrm{L}}, \forall t \in \mathcal{T}, k \in \mathcal{K}, \\
& 0 \leq p_{k}^{\mathrm{U}}(t) \leq P_{k}^{\mathrm{U}}, \forall t \in \mathcal{T}, k \in \mathcal{K}, \\
& 0 \leq \sum_{k \in \mathcal{K}} \psi_{k}(t) \leq 1, \forall t \in \mathcal{T}, \\
& 0 \leq s_{k}(t) \leq S_{k}, \forall t \in \mathcal{T}, k \in \mathcal{K}, \\
& D_{k}(t) \geq A_{k}(t), \forall t \in \mathcal{T}, k \in \mathcal{K},
\end{aligned}
$$

where (7a) is the local CPU frequency constraint, (7b) and (7c) are the transmit power constraints on licensed and unlicensed channels, (7d) is the bandwidth-utilization constraint of the licensed channel, (7e) is to ensure the harmonious coexistence of vehicles and the WiFi users on the unlicensed channels, and (7f) is to ensure that tasks can be processed in a timely manner within the time slot in which they arrive.

As the objective function and constraint (7f) are non-convex, the problem cannot be solved directly using some conventional convex optimisation methods. In the next section, we transform this problem into a convex optimisation problem and then propose a low-complexity lightweight algorithm to obtain optimal offloading and resource allocation scheme.

\section{Joint resource allocation scheme of computing and communication}

\subsection{Problem reformulation}

Although task offloading over unlicensed spectrum is a powerful complement to that via licensed spectrum, the consideration of offloading on unlicensed spectrum introduces 
additional optimization variables, such as the time fraction occupied by each vehicle and the transmit power on unlicensed channels. As a result, the wireless communication scenario considered becomes complex and the corresponding problem becomes non-convex.

To break this barrier, we introduce a new variable $\gamma_{k}(t)$ defined as $\gamma_{k}(t)=p_{k}^{U}(t) s_{k}(t)$. By replacing $p_{k}^{\mathrm{U}}(t)$ with $\frac{\gamma_{k}(t)}{s_{k}(t)}$, problem (7) turns out to be convex, as described in the following process.

Firstly, based on the above replacement, the objective function of problem (20) becomes linear. Secondly, we define a function as

$$
f(x, y)=-C_{1} y \log _{2}\left(1+\frac{C_{2} x}{y}\right)
$$

where $C_{1}$ and $C_{2}$ are constants. Through the positive definiteness of the Hessian matrix, it has been proved to be a convex one. Then, by replacing $p_{k}^{\mathrm{U}}(t)$ with $\frac{\gamma_{k}(t)}{s_{k}(t)}$, (5) and (7f) are transformed separately into

$$
\begin{aligned}
& D_{k}^{\mathrm{U}}(t)=s_{k}(t) B_{k}^{\mathrm{U}} \tau \log _{2}\left(1+\frac{\gamma_{k}(t) h_{k}^{\mathrm{U}}(t)}{N_{0}^{\mathrm{U}} s_{k}(t) B_{k}^{\mathrm{U}}}\right), \\
& 0 \leq \gamma_{k}(t) \leq P_{k}^{\mathrm{U}} S_{k} .
\end{aligned}
$$

It is not difficult to identify that $D_{k}^{\mathrm{U}}(t)$ can be expressed as

$$
D_{k}^{\mathrm{U}}(t)=-f\left(\gamma_{k}(t), s_{k}(t)\right) .
$$

Therefore, constraints in (7) comprise a convex set and the problem now becomes a convex one, which can be solved by Lagrangian dual method [41].

The Lagrange dual function of (7) at time slot $t$ can be written as

$$
\begin{aligned}
\mathcal{L}(\mathbf{X}(t), \lambda(t), \eta(t))= & \sum_{k \in \mathcal{K}}\left(E_{k}^{\text {local }}(t)+E_{k}^{\mathrm{L}}(t)+E_{k}^{\mathrm{U}}(t)\right) \\
& +\eta(t)\left(\sum_{k \in \mathcal{K}} \psi_{k}(t)-1\right)+\sum_{k \in \mathcal{K}} \lambda_{k}(t)\left(A_{k}(t)-D_{k}(t)\right),
\end{aligned}
$$

where $\eta(t), \lambda_{k}(t)$ are the Lagrangian multipliers associated with constraint (7d) and (7f), respectively, and $\lambda(t)$ is the corresponding Lagrange multiplier vector. Thus, the Lagrange dual problem can be expressed as

$$
\begin{aligned}
& \max _{\lambda(t), \eta(t)} \inf _{\mathbf{X}(t) \in \boldsymbol{\Omega}(t)} \mathcal{L}(\mathbf{X}(t), \lambda(t), \eta(t)) \\
& \text { s.t. } \quad \lambda_{k}(t) \geq 0, \forall t \in \mathcal{T}, k \in \mathcal{K}, \\
& \eta(t) \geq 0, \forall t \in \mathcal{T},
\end{aligned}
$$

where $\boldsymbol{\Omega}$ is the feasible region of $\mathbf{X}(t)$ under the constraints from (7a) to (7f). 


\subsection{A low-complexity lightweight algorithm}

Lagrange duality offers an effective method to decouple constraint (7f) and divide problem (7). The Lagrangian dual problem in (13) can be solved optimally by solving the following sub-problems.

\subsubsection{Local computing resource allocation}

Given the fixed $\lambda(t)$, the first sub-problem is to minimize the total energy consumption of all vehicles for local computing in time slot $t$, which can be mathematically written as

$$
\begin{array}{ll}
\min _{\mathbf{f}(t)} & \sum_{k \in \mathcal{K}}\left(E_{k}^{\text {local }}(t)-\lambda_{k}(t) D_{k}^{\text {local }}(t)\right), \\
\text { s.t. } & 0 \leq f_{k}(t) \leq F_{k}, \forall k \in \mathcal{K} .
\end{array}
$$

Each vehicle only needs to use its local computing resources wisely, without sharing local computing information from other vehicles. Hence, the optimisation problem is split up again. The optimal frequency $f_{k}^{*}(t)$ for the local CPU of vehicle $k$ in time slot $t$ can be obtained by solving the following sub-problem :

$$
\begin{aligned}
& \min _{f_{k}(t)} E_{k}^{\text {local }}(t)-\lambda_{k}(t) D_{k}^{\text {local }}(t), \\
& \text { s.t. } \quad 0 \leq f_{k}(t) \leq F_{k} .
\end{aligned}
$$

Problem (15) is a convex optimization problem, and thus, the Karush-Kuhn-Tucker (KKT) conditions can be used to find the optimal solution. In this problem, we can obtain the first-order derivative with respect to local frequency as

$$
\frac{\partial \mathcal{L}}{\partial f_{k}(t)}=3 \tau \mu_{k}\left(f_{k}(t)\right)^{2}-\frac{\lambda_{k}(t) \tau}{M_{k}(t)}
$$

Setting $\frac{\partial \mathcal{L}}{\partial f_{k}(t)}=0$, we obtain the optimal CPU frequency of vehicle $k$ in time slot $t$ as

$$
f_{k}^{*}(t)=\left[\sqrt{\frac{\lambda_{k}(t)}{3 \mu_{k} M_{k}(t)}}\right]_{0}^{F_{k}},
$$

where $[a]_{x}^{y}=\min (y, \max (a, x))$.

\subsubsection{Licensed communication resource allocation}

Given the fixed $\lambda(t)$ and $\eta(t)$, the second sub-problem is to minimize the total energy consumption of all vehicles for offloading through the licensed channels. Therefore, the optimal transmit power $p_{k}^{L *}(t)$ and proportion of licensed bandwidth $\psi_{k}^{*}(t)$ in time slot $t$ can be obtained by solving the following sub-problem:

$$
\min _{\mathbf{p}^{\mathrm{L}}(t), \psi(t),} \sum_{k \in \mathcal{K}}\left(E_{k}^{\mathrm{L}}(t)-\lambda_{k}(t) D_{k}^{\mathrm{L}}(t)\right)+\eta(t)\left(\sum_{k \in \mathcal{K}} \psi_{k}(t)-1\right),
$$




$$
\text { s.t. } \quad 0 \leq p_{k}^{\mathrm{L}}(t) \leq P_{k}^{\mathrm{L}}, \forall k \in \mathcal{K} .
$$

Considering the high complexity of general convex optimization algorithms, we solve (18) by optimizing the transmit power and the bandwidth allocation in an alternating manner, which is guaranteed to converge to the global optimal solution with a sublinear convergence rate [42]. Hence, we take the first-order derivatives of Lagrange dual function with respect to $p_{k}^{\mathrm{L}}(t)$ and $\psi_{k}(t)$ as

$$
\begin{aligned}
& \frac{\partial \mathcal{L}}{\partial p_{k}^{\mathrm{L}}(t)}=\tau-\frac{\tau \lambda_{k}(t) h_{k}^{\mathrm{L}}(t) B^{\mathrm{L}} \psi_{k}(t)}{\ln 2\left(N_{0}^{\mathrm{L}} B^{\mathrm{L}} \psi_{k}(t)+p_{k}^{\mathrm{L}}(t) h_{k}^{\mathrm{L}}(t)\right)}, \\
& \frac{\partial \mathcal{L}}{\partial \psi_{k}(t)}=\eta(t)-\lambda_{k}(t)\left[\frac{D_{k}^{\mathrm{L}}(t)}{\psi_{k}(t)}-\frac{\tau p_{k}^{\mathrm{L}}(t) h_{k}^{\mathrm{L}}(t) B^{\mathrm{L}}}{\ln 2\left(N_{0}^{\mathrm{L}} B^{\mathrm{L}} \psi_{k}(t)+p_{k}^{\mathrm{L}}(t) h_{k}^{\mathrm{L}}(t)\right)}\right] .
\end{aligned}
$$

In each iteration, the optimal transmit power and bandwidth allocated to vehicle $k$ are achieved at either the stationary point of (19) and (20) or one of the boundary points, which are given by

$$
\begin{aligned}
& p_{k}^{\mathrm{L} *}(t)=\left[\psi_{k}(t) B^{\mathrm{L}}\left(\frac{\lambda_{k}(t)}{\ln 2}-\frac{N_{0}^{\mathrm{L}}}{h_{k}^{\mathrm{L}}(t)}\right)\right]_{0}^{P_{k}^{\mathrm{L}}}, \\
& \psi_{k}^{*}(t)=\left[\tilde{\psi}_{k}^{*}(t)\right]_{0}^{1}
\end{aligned}
$$

where $\tilde{\psi}_{k}^{*}(t)$ satisfies the following equation.

$$
\log _{2}\left(1+\frac{1}{X}\right)-\frac{1}{(1+X) \ln 2}=\frac{\eta(t)}{\lambda_{k}(t) B^{\mathrm{L}} \tau}
$$

where $X=\frac{\tilde{\psi}_{k}^{*}(t) N_{0}^{\mathrm{L}} B^{\mathrm{L}}}{h_{k}^{\mathrm{L}}(t) p_{k}^{\mathrm{L}}(t)}$.

\subsubsection{Unlicensed communication resource allocation}

Given the fixed $\lambda(t)$ and $\eta(t)$, the third sub-problem is to minimize the total energy consumption of all vehicles for offloading through the unlicensed channels in time slot $t$, which can be mathematically written as

$$
\begin{aligned}
& \min _{\boldsymbol{\gamma}(t), \mathbf{s}(t),} \quad \sum_{k \in \mathcal{K}}\left(E_{k}^{\mathrm{U}}(t)-\lambda_{k}(t) D_{k}^{\mathrm{U}}(t)\right), \\
& \text { s.t. } \quad 0 \leq \gamma_{k}(t) \leq P_{k}^{\mathrm{U}} S_{k}, \forall k \in \mathcal{K}, \\
& 0 \leq s_{k}(t) \leq S_{k}, \forall k \in \mathcal{K},
\end{aligned}
$$

where $\boldsymbol{\gamma}(t) \triangleq\left\{\gamma_{1}(t), \gamma_{2}(t), \ldots, \gamma_{K}(t)\right\}$.

As each vehicle only needs to share an unlicensed channel with nearby WiFi users without competing with other vehicles, the problem can be also split into a per-vehicle 
optimisation sub-problem. On the basis, the optimal transmit power $p_{k}^{\mathrm{U} *}(t)$ and the optimal time fraction $s_{k}^{*}(t)$ on the unlicensed channel can be obtained by solving the following sub-problem:

$$
\begin{aligned}
& \min _{\gamma_{k}(t), s_{k}(t),} \quad E_{k}^{\mathrm{U}}(t)-\lambda_{k}(t) D_{k}^{\mathrm{U}}(t) \\
& \text { s.t. } \quad 0 \leq \gamma_{k}(t) \leq P_{k}^{\mathrm{U}} S_{k}, \\
& 0 \leq s_{k}(t) \leq S_{k} .
\end{aligned}
$$

Since the objective function of (25) is non-increasing with respect to $s_{k}(t)$, for any $k \in \mathcal{K}$, the optimal time fraction occupied by vehicle $k$ on unlicensed channel is equal to the maximum value, i.e. $s_{k}^{*}(t)=S_{k}$.

Similarly, for offloading tasks on unlicensed channels, we have

$$
\gamma_{k}^{*}(t)=\left[S_{k} B_{k}^{\mathrm{U}}\left(\frac{\lambda_{k}(t)}{\ln 2}-\frac{N_{0}^{\mathrm{U}}}{h_{k}^{\mathrm{U}}(t)}\right)\right]_{0}^{P_{k}^{\mathrm{U}} S_{k}} .
$$

Therefore, the optimal transmit power $p_{k}^{\mathrm{U} *}(t)$ is achieved at either the stationary point of (25) or one of the boundary points, which is given by

$$
p_{k}^{\mathrm{U} *}(t)=\frac{\gamma_{k}^{*}(t)}{s_{k}^{*}(t)}=\left[B_{k}^{\mathrm{U}}\left(\frac{\lambda_{k}(t)}{\ln 2}-\frac{N_{0}^{\mathrm{U}}}{h_{k}^{\mathrm{U}}(t)}\right)\right]_{0}^{P_{k}^{\mathrm{U}}} .
$$

\subsubsection{Optimal Lagrange multiplier}

Once the optimal resource allocation is achieved by (17), (21), (22), (27), the solution of dual problem (13) can be determined by a subgradient method as follows [43]:

$$
\begin{aligned}
& \lambda_{k}^{(i+1)}(t)=\left[\lambda_{k}^{(i)}(t)+\varsigma_{\lambda}\left(A_{k}(t)-D_{k}(t)\right)\right]^{+}, \\
& \eta^{(i+1)}(t)=\left[\eta^{(i)}(t)+\varsigma_{\eta}\left(\sum_{k \in \mathcal{K}} \psi_{k}(t)-1\right)\right]^{+},
\end{aligned}
$$

where $i$ is the iteration index, and $\varsigma_{\lambda}$ and $\varsigma_{\eta}$ are the positive step sizes.

The Lagrange multipliers $\lambda(t)$ and $\eta(t)$ can be independently updated by (28) and (29), respectively. The update process will be repeated until convergence, indicating that the dual optimal point has been reached. Since the joint power, spectrum and computing resource allocation problem proves to be convex, the duality gap can be guaranteed to zero according to $[43,44]$. Algorithm 1 briefly summarizes the proposed algorithm. 


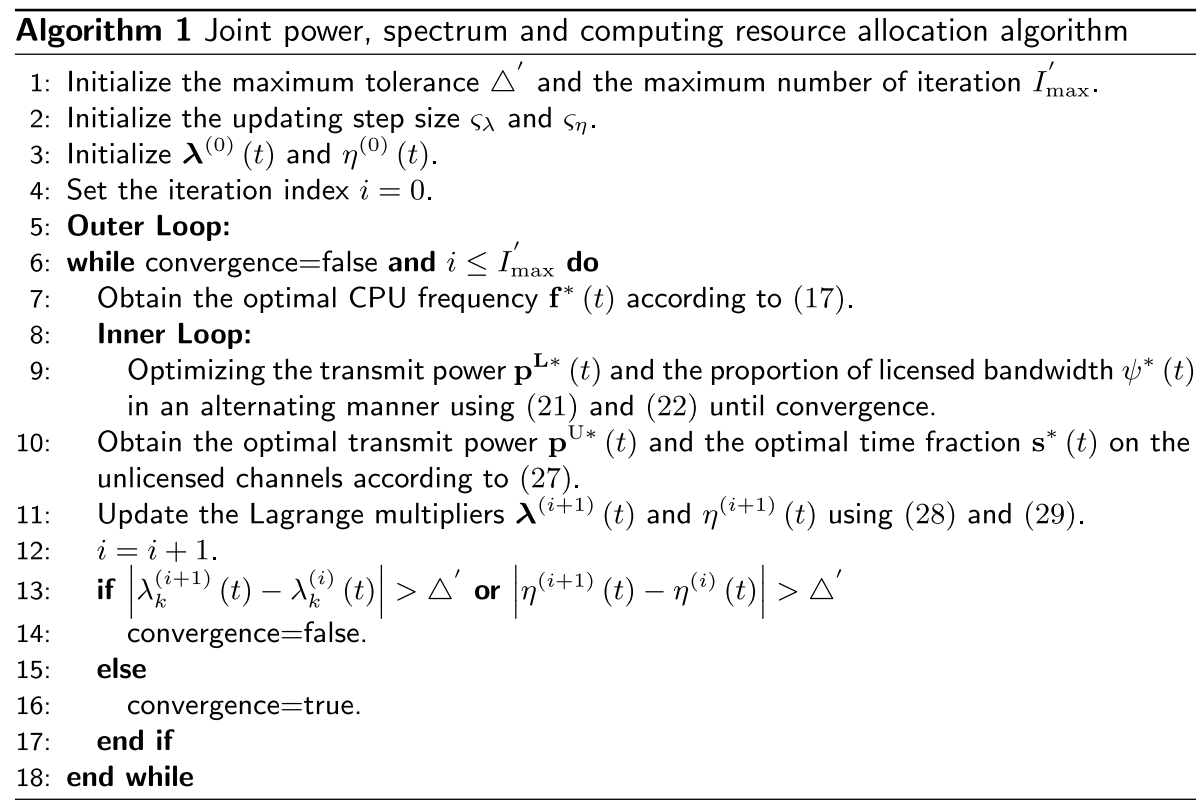

\subsection{Algorithm complexity analysis}

In each time slot, the computational complexity of the proposed scheme mainly lies in both loops shown in Algorithm 1. For the inner loop that determines the proportion of tasks offloaded on the licensed channels, alternately updating the transmit power and proportion of licensed bandwidth has been shown in [42] to converge to the optimal solution with a sublinear convergence rate. Besides, the outer loop is a standard convex optimization and a fast convergence speed can be guaranteed for the subgradient method. As will be demonstrated in the simulation, only 6-9 iterations are required for the Lagrange multipliers to converge. Therefore, we can qualitatively conclude that the computational complexity of the proposed algorithm is low and acceptable.

\section{Simulation results and discussion}

In this section, simulation results are presented to verify the efficiency of the proposed algorithm. We consider a single-cell system with a radius of $200 \mathrm{~m}$, where the RSU is located at the centre of the cell and vehicles are uniformly distributed in the cell. The distance between vehicle $k$ and the RSU is denoted by $D_{k}$. In addition, there are a certain number of WiFi users distributed around each vehicle. Each vehicle adopts the DCM mechanism to share the unlicensed channel with the associated WiFi users. In the simulations, the channels between vehicles and the RSU are assumed to be frequency-flat. The major parameters used in our simulation are listed in Table 1.

\subsection{System performance}

To demonstrate the necessity of offloading on unlicensed spectrum, we set the simulation environment to three scenarios, including a VEC network that only uses licensed access, a VEC network that only uses unlicensed access and a VEC network that 
Table 1 Simulation parameter setting

\begin{tabular}{ll}
\hline Parameter & Value \\
\hline Radius of the cell & $200 \mathrm{~m}$ \\
Time slot & $1 \mathrm{~ms}$ \\
Pass loss model (licensed, unlicensed) & $15.3+10 \sigma \log _{10}\left(D_{k}\right)$, \\
Noise power spectral density $N_{0}^{L}, N_{0}^{\cup}$ & $\sigma=3.75,5$ \\
Maximum transmit power on licensed channels $P_{k}^{L}$ & $-174 \mathrm{dBm} / \mathrm{Hz}$ \\
Maximum transmit power on unlicensed channels $P_{k}^{U}$ & $500 \mathrm{~mW}$ \\
Effective switched capacitance of CPU $\mu_{k}$ & $200 \mathrm{~mW}$ \\
Processing density request $M_{k}$ & $10^{-27}$ \\
Maximum CPU frequency $F_{k}$ & $100 \mathrm{cycles} / \mathrm{bit}$ \\
\hline
\end{tabular}

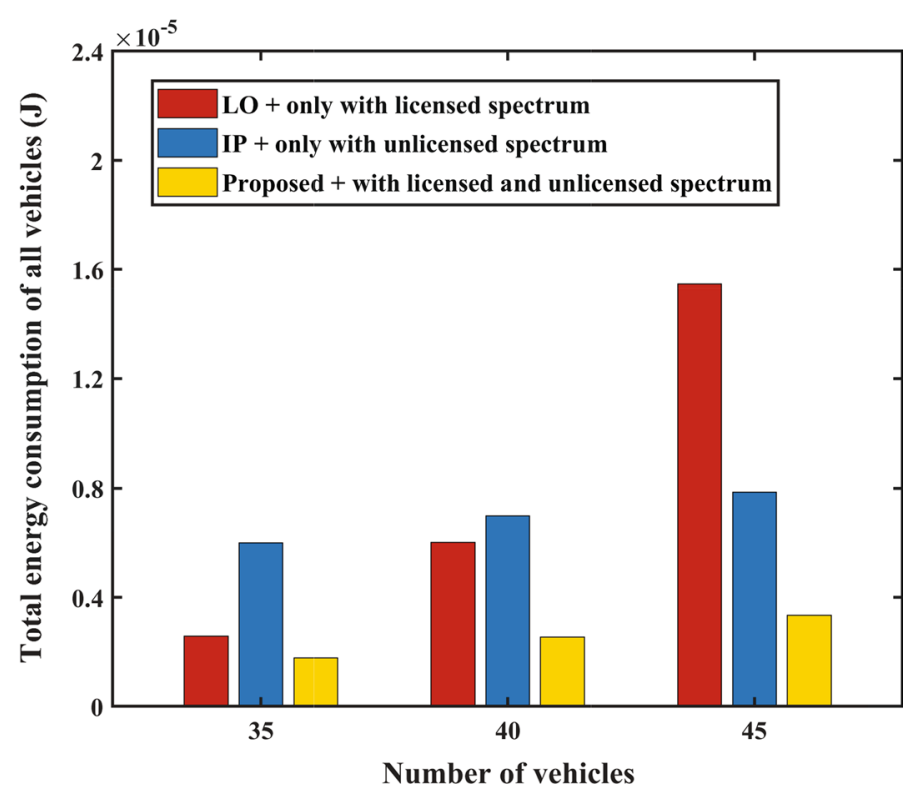

Fig. 2 The total energy consumption of all vehicles versus the number of vehicles, $B^{L}=20 \mathrm{MHz}, S_{k}=0.5$, and $B_{k}^{\bigcup}=20 \mathrm{MHz}$ for all $k \in \mathcal{K}$

employs licensed and unlicensed access jointly. Corresponding to the first two scenarios, we use the following two schemes as baselines. The first baseline is a centralized Lyapunov optimisation (LO)-based algorithm, which is applied to the scenario that vehicles can only process tasks through local computing and offloading to the RSU through licensed channels. Considering the novelty of introducing unlicensed spectrum to the VEC networks, we use the centralized interior point (IP) method as the second baseline. The IP algorithm is applied under the scenario, where vehicles can only process tasks through local computing and offloading to the RSU through unlicensed channels.

Figure 2 shows the energy consumption performance of three algorithms with varying number of vehicles. We can observe that the energy consumption in the third scenario is always lower than that in the first two scenarios. The reason is that joint employing 
licensed and unlicensed access in the VEC network lead to the expansion of the spectrum resources available. At the same data rate, more spectrum resources will result in less transmit power. In particular, the gap in energy consumption between the scenario only with licensed spectrum and the scenario with two spectrums widens as the number of vehicles increases. When the licensed spectrum is not enough to offload the tasks of vehicles, the tasks have to be processed locally by the LO algorithm, which consumes more energy.

\subsection{The convergence of the proposed algorithm}

Figure 3 shows the convergence on the energy consumption of the vehicles when the proposed algorithm is applied. The iterative process illustrated in the figure is consistent with the update process of the Lagrange multipliers in the outer loop in Algorithm 1. Obviously, the proposed algorithm requires only 6-9 external iterations to obtain the optimal Lagrange multipliers. This finding provides the proof for the theoretical analysis about the algorithm complexity in Sect. 5.3. For actual road sections with different traffic volumes, we can set different step sizes to ensure the fastest convergence rate.

\subsection{The impact of licensed spectrum resource}

Figure 4a shows the impact of licensed spectrum bandwidth on the amount of offloaded tasks. Obviously, numerous vehicles and low licensed bandwidth imply that the licensed spectrum resource is scarce. In this case, tasks that would have been offloaded via licensed channels are transferred to unlicensed channels. In contrast, when the licensed spectrum resources are enough, task offloading would be only performed on the licensed channel since it does not need to consume energy for sense the channel state. Besides, an increase on spectrum resources will result in a decrease

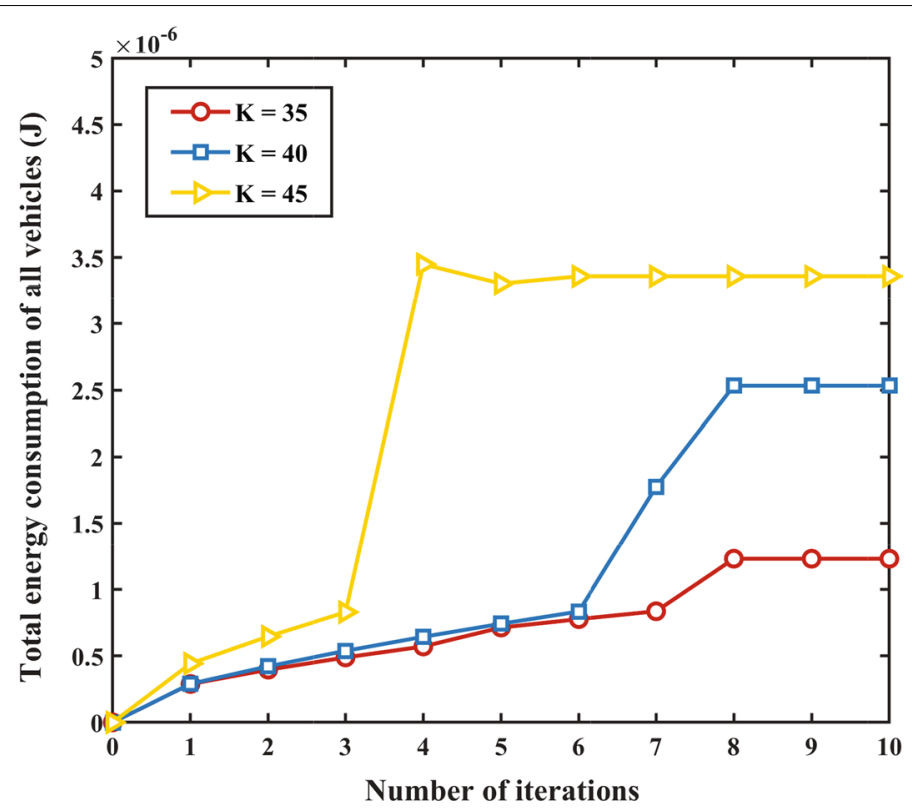

Fig. 3 Convergence behaviour for the total energy consumption of all vehicles versus the number of vehicles, $B^{L}=20 \mathrm{MHz}, S_{k}=0.5$, and $B_{k}^{\cup}=20 \mathrm{MHz}$ for all $k \in \mathcal{K}$ 


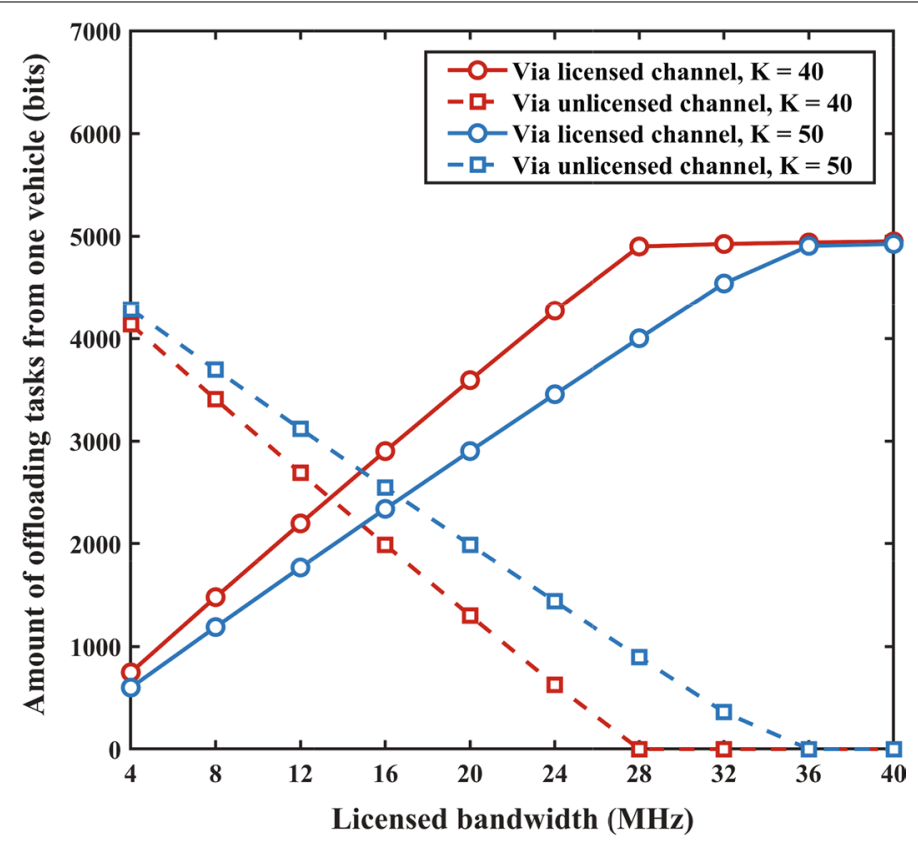

(a)

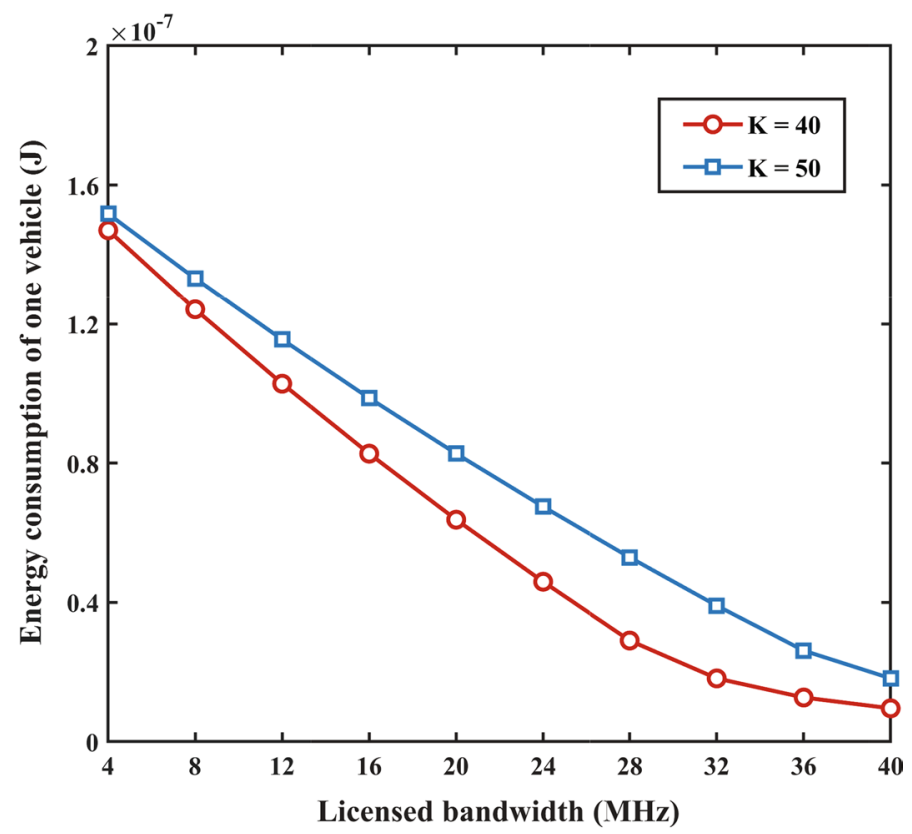

(b)

Fig. 4 The impact of licensed spectrum bandwidth, $S_{k}=0.5$, and $B_{k}^{U}=20 \mathrm{MHz}$ for all $k \in \mathcal{K}$ : a the effect on task offloading from one vehicle; $\boldsymbol{b}$ the effect on energy consumption of one vehicle

on energy consumption as demonstrated in Fig. 4b since more task bits can be offloaded to the MEC server via the licensed channels. Due to the lower path loss on licensed channels, the energy can be used more efficiently on the licensed spectrum. Therefore, the trends in these two subplots are partially approximately linear. 


\subsection{The impact of unlicensed spectrum resource}

Figure 5 shows the impact of the available time fraction of unlicensed channel at vehicle $k, S_{k}$, on the task offloading and energy consumption with different unlicensed bandwidths. As shown in Fig. 5a, the amount of tasks offloaded on the unlicensed channel increases as the available unlicensed spectrum resource increases. However,

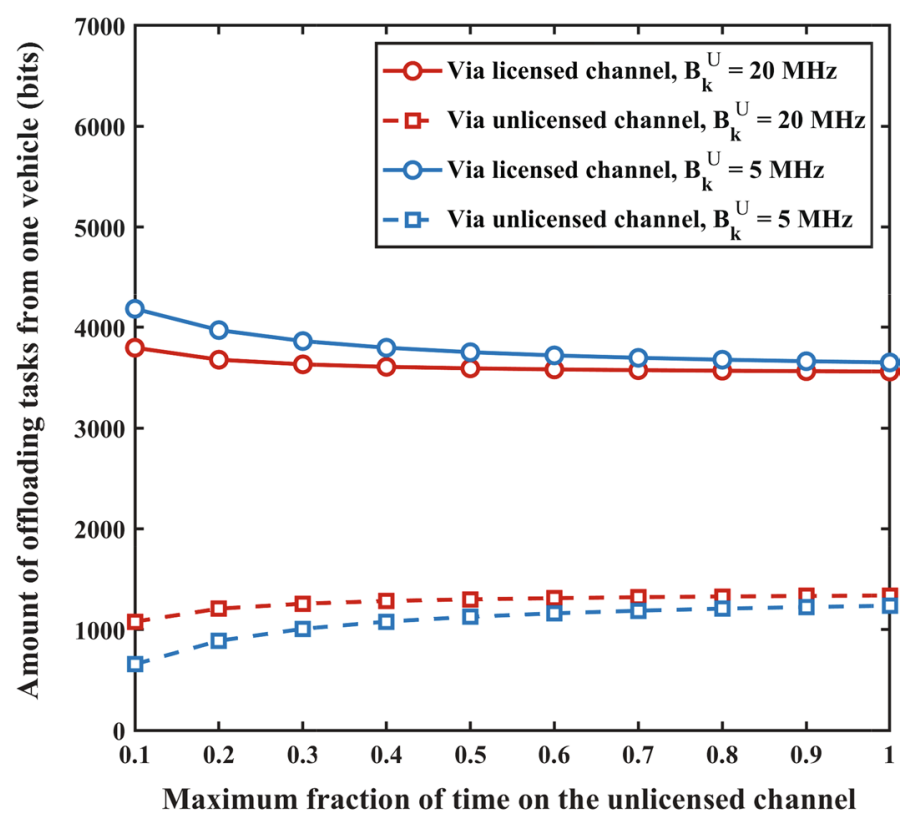

(a)

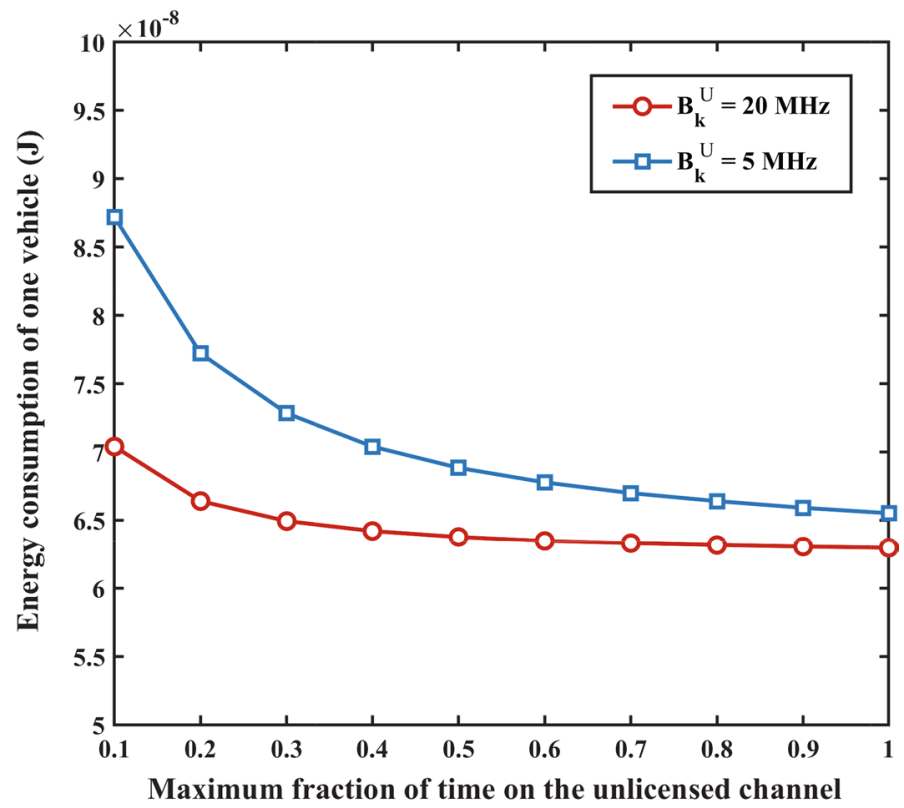

(b)

Fig. 5 The effect of available time fraction on unlicensed channels on the task offloading and energy consumption at the vehicle, $K=40, B^{L}=20 \mathrm{MHz}$ : $\mathbf{a}$ the impact on task offloading from one vehicle; $\mathbf{b}$ the impact on energy consumption of one vehicle 
when licensed spectrum resources are relatively plentiful, the change in the strategy of offloading tasks is relatively flat. On the other hand, Fig. 5b shows the dramatic impact on energy consumption despite the slight change in task allocation ratio, demonstrating the important role of unlicensed spectrum in VEC networks.

\title{
7 Conclusion
}

In this paper, employing licensed and unlicensed access in the VEC networks makes the strategies for offloading tasks more complicated. To solve the joint power, spectrum and computing resource allocation problem, we propose a low-complexity algorithm to minimize the total energy consumption of all vehicles for processing tasks. Theoretical analysis and simulation results validate the effectiveness of the proposed scheme that the additional introduction of unlicensed spectrum effectively can effectively reduce the energy consumption in comparison with baseline schemes. In future works, we will extend the findings in this work to multi-RSU multi-cell VEC scenarios and explore distributed implementation methods.

\begin{abstract}
Abbreviations
MEC: Mobile Edge Computing; VEC: Vehicular Edge Computing; ITS: Intelligent Transportation System; AR: Augmented Reality; FCC: Federal Communications Commission; DSRC: Dedicated Short-Range Communications; ALTO: Adaptive Learning-based Task Offloading; QoE: Quality of Experience; CVEC: Collaborative Vehicular Edge Computing; IloT: Industrial Internet of Things; NOMA: Non-orthogonal Multiple Access; loT: Internet of Things; 5G: Fifth Generation; WIT: Wireless Information Transfer; WPT: Wireless Power Transfer; MSS: Multibeam Satellite Systems; V2X:Vehicle-to-Everything; VANET: Vehicular Ad hoc Network; QoS: Quality of Services; V2I: Vehicle-to-Infrastructure; V2R: Vehicle-to-Road-side unit; RSU: Road-Side Unit; FDMA: Frequency Division Multiple Access; DCM: Duty-Cycle Muting; TDMA: Time Division Multiple Access; KKT: Karush-Kuhn-Tucker.
\end{abstract}

Acknowledgements

Not applicable.

\section{Author Contributions}

ZJ conceived of the presented idea and wrote the manuscript. ZJ, XL and RY developed the theory and scheme. CW verified the analytical methods and checked the manuscript. All authors read and approved the final manuscript.

Funding

This work was supported in part by the National Natural Science Foundation of China (Grant No. 61771429), in part by ROIS NII Open Collaborative Research 2150601 and in part by JSPS KAKENHI (Grant Nos. 18KK0279, 19H04093, $20 \mathrm{H00592}$ and 21H03424).

Availability of data and materials

The data included in this paper are available without any restriction.

\section{Declarations}

Ethics approval and consent to participate

Not applicable.

\section{Consent for publication}

The manuscript does not contain any individual person's data in any form (including individual details, images or videos), and therefore, the consent for publication is not applicable to this article.

\section{Competing interests}

The authors declare that they have no competing interests.

\section{Author details}

${ }^{1}$ School of Intelligent Manufacturing, Wenzhou Polytechnic, Wenzhou, China. ${ }^{2}$ School of Information and Electronic Engineering, Zhejiang University, Hangzhou, China. ${ }^{3}$ School of Information and Electrical Engineering, Zhejiang University City College, Hangzhou, China. ${ }^{4}$ Graduate School of Informatics and Engineering, University of Electro-Communications, Tokyo, Japan. 


\section{References}

1. L. Qi, Research on intelligent transportation system technologies and applications. 2008 Workshop on Power Electronics and Intelligent Transportation System (IEEE, 2008), pp. 529-531

2. Y. Lin, P. Wang, M. Ma, Intelligent transportation system (ITS): concept, challenge and opportunity. 2017 IEEE 3rd International Conference on Big Data Security on Cloud (BigDataSecurity), IEEE International Conference on High Performance and Smart Computing (HPSC), and IEEE International Conference on Intelligent Data and Security (IDS) (IEEE, 2017), pp. 167-172

3. S. An, B.H. Lee, D.R. Shin, A survey of intelligent transportation systems. 2011 Third International Conference on Computational Intelligence, Communication Systems and Networks (IEEE, 2011), pp. 332-337

4. Z. Lu, G. Qu, Z. Liu, A survey on recent advances in vehicular network security, trust, and privacy. IEEE Trans. Intell. Transp. Syst. 20(2), 760-776 (2018)

5. J. Levinson, J. Askeland, J. Becker, J. Dolson, D. Held, S. Kammel, Towards fully autonomous driving: systems and algorithms. 2011 IEEE Intelligent Vehicles Symposium (IV) (IEEE, 2011), pp. 163-168

6. S. Tachi, M. Inami, Y. Uema, Augmented reality helps drivers see around blind spots. IEEE Spectrum 31 (2014)

7. M. Whaiduzzaman, M. Sookhak, A. Gani, R. Buyya, A survey on vehicular cloud computing. J. Netw. Comput. Appl. 40, 325-344 (2014)

8. S. Olariu, I. Khalil, M. Abuelela, Taking VANET to the clouds. Int. J. Pervasive Comput. Commun. 7(1), 7-21 (2011)

9. M. Chiang, T. Zhang, Fog and IoT: an overview of research opportunities. IEEE Internet Things J. 3(6), 854-864 (2016)

10. S. Raza, S. Wang, M. Ahmed, M.R. Anwar, A survey on vehicular edge computing: architecture, applications, technical issues, and future directions. Wirel. Commun. Mobile Comput. 2019, 3159762 (2019)

11. X. Hou, Y. Li, M. Chen, D. Wu, D. Jin, S. Chen, Vehicular fog computing: a viewpoint of vehicles as the infrastructures. IEEE Trans. Veh. Technol. 65(6), 3860-3873 (2016)

12. L. Liu, C. Chen, Q. Pei, S. Maharjan, Y. Zhang, Vehicular edge computing and networking: a survey. Mobile Netw. Appl. 26, 1145-1168 (2020)

13. J.B. Kenney, Dedicated short-range communications (DSRC) standards in the United States. Proc. IEEE 99(7), $1162-1182(2011)$

14. US Federal Communications Commission et al., Dedicated short range communications report and order (R\&O FCC, 2003), pp. 3-324

15. Y. Chen, N. Zhang, Y. Zhang, X. Chen, W. Wu, X.S. Shen, Energy efficient dynamic offloading in mobile edge computing for Internet of Things. IEEE Trans. Cloud Comput. 9(3), 1050-1060 (2019)

16. Y. Mao, J. Zhang, S. Song, K.B. Letaief, Stochastic joint radio and computational resource management for multi-user mobile-edge computing systems. IEEE Trans. Wirel. Commun. 16(9), 5994-6009 (2017)

17. C.F. Liu, M. Bennis, M. Debbah, H.V. Poor, Dynamic task offloading and resource allocation for ultra-reliable lowlatency edge computing. IEEE Trans. Commun. 67(6), 4132-4150 (2019)

18. J. Guo, Z. Song, Y. Cui, Z. Liu, Y. Ji, Energy-efficient resource allocation for multi-user mobile edge computing. GLOBECOM 2017-2017 IEEE Global Communications Conference (IEEE, 2017), pp. 1-7

19. T. Mahn, H. Al-Shatri, A. Klein, Distributed algorithm for energy efficient joint cloud and edge computing with splittable tasks. 2019 IEEE Wireless Communications and Networking Conference (WCNC) (IEEE, 2019), pp. 1-7

20. C. Wu, Z. Liu, F. Liu, T. Yoshinaga, Y. Ji, J. Li, Collaborative learning of communication routes in edge-enabled multiaccess vehicular environment. IEEE Trans. Cogn. Commun. Netw. 6(4), 1155-1165 (2020)

21. Y. Sun, X. Guo, J. Song, S. Zhou, Z. Jiang, X. Liu et al., Adaptive learning-based task offloading for vehicular edge computing systems. IEEE Trans. Veh. Technol. 68(4), 3061-3074 (2019)

22. S. Raza, W. Liu, M. Ahmed, M.R. Anwar, M.A. Mirza, Q. Sun et al., An efficient task offloading scheme in vehicular edge computing. J. Cloud Comput. 9, 1-14 (2020)

23. Z. Ning, P. Dong, X. Wang, J.J. Rodrigues, F. Xia, Deep reinforcement learning for vehicular edge computing: an intelligent offloading system. ACM Trans. Intell. Syst. Technol. (TIST) 10(6), 1-24 (2019)

24. J. Sun, Q. Gu, T. Zheng, P. Dong, A. Valera, Y. Qin, Joint optimization of computation offloading and task scheduling in vehicular edge computing networks. IEEE Access 8, 10466-10477 (2020)

25. X. Huang, R. Yu, D. Ye, L. Shu, S. Xie, Efficient workload allocation and user-centric utility maximization for task scheduling in collaborative vehicular edge computing. IEEE Trans. Veh. Technol. 70(4), 3773-3787 (2021)

26. X. Liu, X. Zhang, NOMA-based resource allocation for cluster-based cognitive industrial internet of things. IEEE Trans. Ind. Inform. 16(8), 5379-5388 (2019)

27. X. Liu, X.B. Zhai, W. Lu, C. Wu, QoS-guarantee resource allocation for multibeam satellite industrial Internet of Things with NOMA. IEEE Trans. Ind. Inform. 17(3), 2052-2061 (2019)

28. X. Liu, X. Zhang, Rate and energy efficiency improvements for 5G-based loT with simultaneous transfer. IEEE Internet Things J. 6(4), 5971-5980 (2018)

29. X. Liu, X. Zhang, M. Jia, L. Fan, W. Lu, X. Zhai, 5G-based green broadband communication system design with simultaneous wireless information and power transfer. Phys. Commun. 28, 130-137 (2018)

30. F. Li, K.Y. Lam, X. Liu, J. Wang, K. Zhao, L. Wang, Joint pricing and power allocation for multibeam satellite systems with dynamic game model. IEEE Trans. Veh. Technol. 67(3), 2398-2408 (2017)

31. C. Wu, X. Chen, T. Yoshinaga, Y. Ji, Y. Zhang, Integrating licensed and unlicensed spectrum in the Internet of vehicles with mobile edge computing. IEEE Netw. 33(4), 48-53 (2019)

32. P. Wang, B. Di, H. Zhang, K. Bian, L. Song, Cellular V2X communications in unlicensed spectrum: harmonious coexistence with VANET in 5G systems. IEEE Trans. Wirel. Commun. 17(8), 5212-5224 (2018)

33. T. Xue, H. Zhang, H. Ding, D. Yuan, Vehicle pairing, spectrum assignment and power control for non-safety critical services in heterogeneous vehicular networks. IEEE Trans. Veh. Technol. 70(9), 9160-9173 (2021)

34. Z. Ning, P. Dong, X. Wang, M.S. Obaidat, X. Hu, L. Guo et al., When deep reinforcement learning meets 5G-enabled vehicular networks: a distributed offloading framework for traffic big data. IEEE Trans. Ind. Inform. 16(2), 1352-1361 (2019)

35. T.D. Burd, R.W. Brodersen, Processor design for portable systems. J. VLSI Signal Process. Syst. Signal Image Video Technol. 13(2), 203-221 (1996) 
36. K. De Vogeleer, G. Memmi, P. Jouvelot, F. Coelho, The energy/frequency convexity rule: modeling and experimental validation on mobile devices. International Conference on Parallel Processing and Applied Mathematics (Springer, 2013), pp. 793-803

37. T.M. Cover, Elements of Information Theory (Wiley, Hoboken, NJ, 1999)

38. Qualcomm. Qualcomm research LTE in unlicensed spectrum: harmonious coexistence with Wi-Fi. Technical Report, Qualcomm Technologies, Inc (2014)

39. E. Almeida, A.M. Cavalcante, R.C. Paiva, F.S. Chaves, F.M. Abinader, R.D. Vieira et al., Enabling LTE/WiFi coexistence by LTE blank subframe allocation. 2013 IEEE International Conference on Communications (ICC) (IEEE, 2013), pp. 5083-5088

40. Z. Zou, R. Yin, C. Wu, J. Yuan, X. Chen, Distributed spectrum and power allocation for D2D-U networks International Conference on Mobile Networks and Management (Springer, 2020), pp. 161-180

41. D.P. Palomar, M. Chiang, A tutorial on decomposition methods for network utility maximization. IEEE J. Sel. Areas Commun. 24(8), 1439-1451 (2006)

42. L. Grippo, M. Sciandrone, On the convergence of the block nonlinear Gauss-Seidel method under convex constraints. Oper. Res. Lett. 26(3), 127-136 (2000)

43. S. Boyd, L. Xiao, A. Mutapcic, Subgradient Methods. Lecture Notes of EE3920, Vol. 2003 (Stanford University, Autumn Quarter, 2004), pp. 2004-2005

44. S. Boyd, S.P. Boyd, L. Vandenberghe, Convex Optimization (Cambridge University Press, Cambridge, 2004)

\section{Publisher's Note}

Springer Nature remains neutral with regard to jurisdictional claims in published maps and institutional affiliations.

\section{Submit your manuscript to a SpringerOpen ${ }^{\circ}$ journal and benefit from:}

- Convenient online submission

- Rigorous peer review

Open access: articles freely available online

- High visibility within the field

Retaining the copyright to your article

Submit your next manuscript at $\gg$ springeropen.com 